\title{
The Opportunity for Smart Charging to Mitigate the Impact of Electric Vehicles on Transmission and Distribution Systems
}

\author{
Constance Crozier ${ }^{\mathrm{a}, *}$, Thomas Morstyn ${ }^{\mathrm{a}}$, Malcolm McCulloch ${ }^{\mathrm{a}}$ \\ ${ }^{a}$ Department of Engineering Science, University of Oxford, Parks Road, OX1 3PJ
}

\begin{abstract}
A rapid increase in the number of electric vehicles is expected in coming years, driven by government incentives and falling battery prices. Charging these vehicles will add significant load to the electricity network, and it is important to understand the impact this will have on both the transmission and distribution level systems, and how smart charging can alleviate it. Here we analyse the effects that charging a large electric vehicle fleet would have on the power network, taking into account the spatial heterogeneity of vehicle use, electricity demand, and network structure. A conditional probability based method is used to model uncontrolled charging demand, and convex optimisation is used to model smart charging. Stochasticity is captured using Monte Carlo simulations. It is shown that for Great Britain's power system, smart charging can simultaneously eliminate the need for additional generation infrastructure required with 100\% electric vehicle adoption, while also reducing the percentage of distribution networks which would require reinforcement from $28 \%$ to $9 \%$. Discussion is included as to how far these results can be extended to other power systems.
\end{abstract}

Keywords: Distribution system, Electric vehicles, Smart charging, Transmission system

\section{Introduction}

This paper quantifies the impact that electric vehicle $(\mathrm{EV})$ charging will have on transmission and distribution systems, and investigates how far smart charging can be used to reduce the impact on both systems.

Falling battery prices and government incentives have led to a rapid increase in sales of electric vehicles $(\mathrm{EVs})$ in the developed world. As of Q4 2019, there were 259,000 EVs on the UK roads [1], and this number is forecast to rise to $36,000,000$ by 2040 [2]. The transition away from internal combustion engines will play a vital role in meeting the decarbonisation targets set in the Paris Climate Change Agreement [3]. However, charging EVs will add large loads to the existing electrical power systems. It is likely that the majority of electric vehicle charging will be done

\footnotetext{
${ }^{*}$ Corresponding author.
}

at home - early observations show home charging accounts for $87 \%$ of charging sessions [4]. This means vehicles will be connected at the grid edge, and therefore will impact the whole electric power system. Domestic EV charging will drastically increase household demand for electricity [5], and it is expected that the existing early evening peak in demand will be exacerbated [6]. This could lead to a variety of problems for the power system, at both the transmission system level [7], and the distribution system level [8].

Electricity supply is commonly divided into three stages: generation, transmission, and distribution. The power generation system encompasses the production of electricity and the allocation of required demand between producers. The transmission system transports the power from its source to grid supply points using high voltage transmission lines. These two systems are often grouped, as they are both concerned with demand 
and supply at the national level, and will be hereafter referred to as the transmission level system. The distribution system connects homes to their nearest grid supply point using a large number of lower voltage circuits. In this paper, the lowest voltage residential circuits in the distribution network are focused on, as the impacts are expected to be worst at this point. The additional demand created by electric vehicles will impact each of these systems in different ways.

At the generation level, the peak demand is likely to increase, which could violate the UK's supply constraints - meaning that, even with maximum power generation, the demand can not be met. This would necessitate installing additional generation capacity, likely in the form of traditional fossil fuel power plants. A change in the demand profile would also affect the mix of fuels that are used, making it more challenging to meet the UK's commitments to increase the percentage of electricity which is generated from renewable sources [3]. The impact on the transmission and distribution systems depends on the locations of the additional load. If the load is concentrated in a particular region, some transmission lines could be forced to carry currents above their rated limits, and the resistive losses in the high-voltage network would increase. Distribution networks with larger amounts of vehicle charging may experience problems. Each network is connected to the higher-voltage system using a transformer that is rated to a specific maximum demand; if this would be exceeded, the transformer must be replaced. Resistive losses will also increase, resulting in a greater voltage drop across the network. Appliance safety requires bus voltages to stay within $\pm 10 \%$ of the unitary voltage [9], so if the voltage drop increases too dramatically then an intervention by the network operator is required.

Vehicle and electricity use varies significantly geographically; employment [10], building structure [11], and public transport access level [12] all affect the behaviour of consumers. Diversity in behaviour has a cancellation effect, resulting in a lower aggregated peak demand than might be expected. However, the behaviour of consumers liv- ing in the same area is likely to be correlated [13], so the local diversity may be less than the national diversity. Therefore, in order to accurately estimate the future load on a system, the spatial heterogeneity of vehicle and electricity use must be taken into account.

A number of studies have been done investigating the effects of domestic EV charging on a single part of the system. At the transmission level, changes to peak demand [14] and energy consumption [15] have been forecast. However, transmission line loading has not been investigated (as this requires the location as well as size of the load to be estimated). At the distribution level, a large number of case studies have been carried out which quantify the effect of EV charging on transformer loading [16], voltages [17], and losses [18] using a single network case study. However, these results can not be extrapolated to represent the impact on the whole distribution system; the variation in network structure, vehicle use, and demand must be accounted for. There have been analyses of sections of distribution network in Queensland, Australia [19] and the San Francisco Bay Area [20] which take into account geographic variation in travel behaviour. However these use computationally and data intensive methods which can not be scaled to a larger distribution system. In addition to not incorporating spatial heterogeneity, none of these studies simultaneously study both levels of the system. Given that the individual studies all use different assumptions and data sources, this makes it difficult to compare the severity of the impact of $\mathrm{EV}$ charging on each level.

It is anticipated that 'smart charging' - coordinated scheduling of the charging time and power of EVs - will be used to reduce the impact of EVs on power systems. The UK Government has announced that all subsidised home charge points must be smart from July 2019 [21]. However, it has not been determined how these chargers will be coordinated.

A variety of algorithms have been proposed for controlling vehicle charging. Many take the form of formal optimisation problems, and can be categorised according to their control hierarchy, objec- 
tive, and the constraints they take into account. Control can either be centralised, where one actor schedules the charging of a group of EVs, or decentralised, where EVs control their own charging in response to a price signal [22]. The proposed objectives include: flattening load [23], minimising losses [24], reducing phase imbalance [25], and maximising the consumption of renewables [26]. Most algorithms include an energy constraint, which ensures that vehicles achieve the appropriate level of charge. However, some include additional constraints - for example on the network operation [27] or the battery temperature [28].

Alternatively, some propose that charging should be optimised through a market mechanism, where flexibility is sold to the system operator. For example, [29] proposes a strategy for bidding in the wholesale market, [30] looks at providing frequency regulation with EVs, and [31] proposes a method for matching markets with contracts for EV smart charging.

Given the volume of different solutions that have been proposed, there is a risk of different actors making opposing requests of the same vehicles. For example, a distribution network operator could request a decrease in charging to prevent local under-voltages, while the transmission system operator tried to increase charging to raise the system frequency. Some smart charging algorithms take this into account, for example [32] includes constraints set by both the transmission and distribution system operators. However, this conflict has not been taken into account in impact assessments, meaning that the optimal operation of both systems is considered independently.

Previous analysis has either investigated the potential for smart charging to reduce peak demand at the transmission system level (e.g. [33]), or to alleviate the need for infrastructure upgrades within local distribution networks (e.g. [34]). Despite the coupling between these system levels, so far they have been considered in isolation. However, in practice a single objective must be chosen for smart charging - so it will not be possible to achieve these optimal scenarios simultaneously. This may have serious implications when it comes to smart charging, because optimising charging for one level of the system may have a detrimental effect on operation at the other level.

This paper addresses the identified gaps in the existing literature in the following ways. Firstly, spatial heterogeneity of vehicle use, electricity demand, and network structure are incorporated into analysis of the impacts of EV charging on the system. Secondly, the scale of the impact of charging on the transmission and distribution level systems are directly compared and the date of necessary intervention is estimated in each case; this is achieved using a consistent set of modelling assumptions for the uncontrolled and controlled charging of EVs. Thirdly, the conflict between the optimal charging schemes for the system levels is quantified, and the degree to which both systems can be protected simultaneously is investigated. This analysis is vital for network operators, policy makers, and researchers to understand the desired action of smart charging from a whole system perspective.

The focus of this paper is on the security of supply in the power system. That is to say, all analysis focuses on the extent to which the additional demand from EVs can be met without requiring network reinforcements. Security of supply is one of the three core dimensions which make up the World Energy Council's definition of energy sustainability - often referred to as the Energy Trilemma [35]. The other two are energy equity (which defines the electricity cost and how fairly it is shared) and environmental sustainability (which covers the carbon intensity of the system). The addition of EV charging would likely impact all three dimensions, however analysis of the second two is out of the scope of this paper.

The remainder of the paper is structured as follows. In Section 2.1 the data sources used to simulate the geographic variation in vehicle use, electricity demand, and network structure are presented. Section 2.2 describes the models used to estimate regional EV penetration and the aggregated charging profiles. In Section 2.3 the frameworks used for transmission level and distribution level simulations is formulated. The results of the analysis is presented in Section 3 - looking first at the transmission level and distribution level sys- 
tems in isolation, then considering the conflict between them. Sections 4 and 5 conclude the paper.

\section{Methodology}

\subsection{Data sources}

A variety of data sets are required in order to analyse the impacts of EV charging on the power system. This section describes the data used to capture geographic variation in vehicle use, existing electricity demand, and network structure. The datasets described are all for the GB case study, so equivalent data would need to be sourced in order to apply this methodology to another power system.

\subsubsection{Vehicle Use}

The impact of EV charging of the grid depends strongly on the timing of the new demand in relation to the existing demand for electricity. Predicting this requires understanding of the amount of energy vehicles are consuming and when they are likely to charge. Two sources of vehicle use data are utilised in this analysis; usage data from a EV trial [36], and a larger set of travel survey data [37]. While the survey data does not include charging information (as it is based on conventional vehicles) the vehicle use captured is representative of the country's travel as a whole whereas the trial data is likely to describe early adopter behaviour.

\subsubsection{Electricity Demand}

Understanding the current use of the electricity system is important to evaluate the impact charging will have. Therefore several sources of data were used, which describe the UK's electricity demand at various levels of aggregation. For national demand, historic real data is available at 5 minute resolution from [38]. A breakdown of the non-commercial and industrial load was inferred using the Elexon standard profiles [39], alongside aggregated annual meter readings from the census areas [40]. Finally, some 1 minute resolution household loads from a UK trial [41] were used to simulate residential networks.

\subsubsection{Networks}

The effect of charging on losses, line loading, and voltages in a network, will be specific to the topology and impedances of that network. In this paper, two network models were used to investigate impact. First, a reduced version of the GB transmission network was utilised which was formulated in [42]. Secondly, three example 3-phase residential networks are used to quantify the effects of vehicle charging on distribution networks, each representing a different network style. The networks were taken from [43], and are shown in Figure 1. They were purposefully selected to represent a typical network from rural, suburban, and urban areas respectively. The blue circular markers show the locations of households, and the black hexagonal marker shows the substation location.

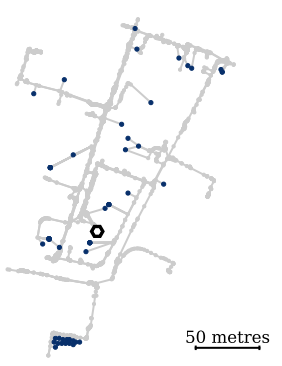

(a) Rural

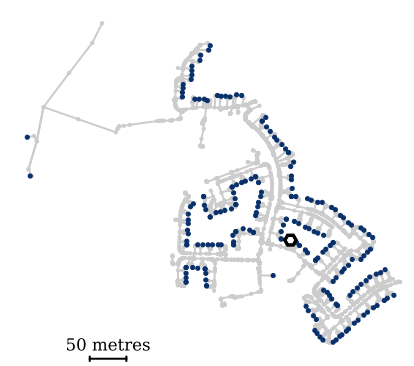

(b) Sub-urban

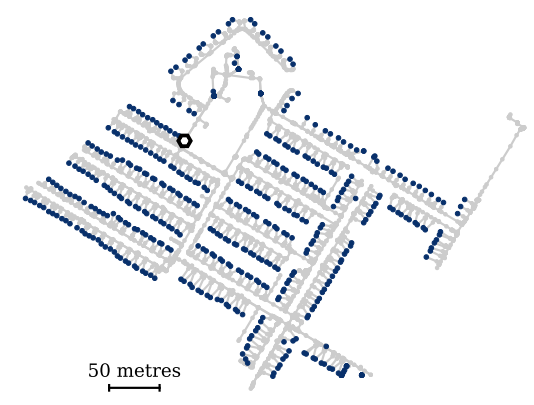

(c) Urban

Figure 1: The test networks used. Blue markers show the household locations and the black marker shows the substation position.

\subsection{Modelling}

This section details the models used to estimate regional EV penetration and the aggre- 
gated charging profiles under both uncontrolled and controlled charging.

\subsubsection{EV Penetration Projection}

Regional vehicle registrations, including the number of ultra low emission vehicles, are published by the Driver and Vehicle Licensing Agency [44]. The percentage of vehicles which were fully electric was assumed to grow in each area according to an s-curve, which are often used to model technological transitions [45]. These curves have the form:

$$
f(x)=\frac{1}{1+\alpha e^{-\beta x}},
$$

where $\alpha, \beta$ are area specific parameters which must be learned from the data. Figure 2a shows an example of the curve fitting process, where the markers show the data and the blue line show the fitted curve. Figure $2 \mathrm{~b}$ then shows the assumed penetration of EVs regionally in 2030 .

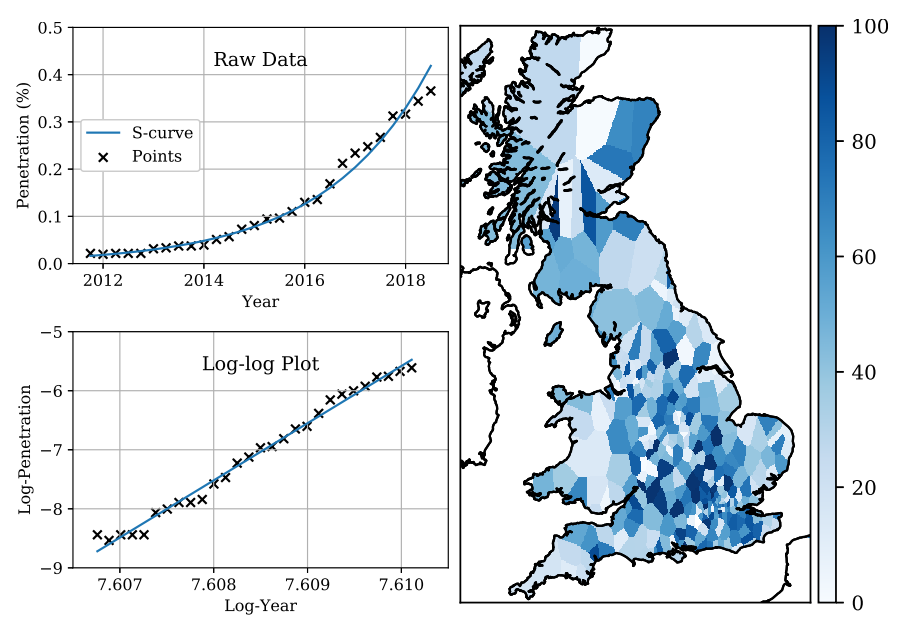

(a) An example s-curve fit

(b) Forecast in $2030(\%)$

Figure 2: Projecting regional EV penetration using scurves.

\subsubsection{Uncontrolled Charging}

Historically, the lack of real-world charging data has meant that simplistic assumptions were made for charging, such as that all vehicles begin charging at once [46], or that charging begins immediately after the completion of the last journey [47]. However, a number of EV charging trials have now been completed in the UK - for example My Electric Avenue [36] and Network Revolution [41] - so higher fidelity modelling techniques are possible. Some research uses trial data directly to estimate the impact of vehicles on the power system [48], however the limited size of the trials mean that they likely capture a biased set of driving. Here the uncontrolled charging scenario is modelled using a stochastic model developed by the authors in [49]. This model essential maps the charging observed from the EV trial data onto the vehicle usage captured in the survey data. This allows the geographic variation in the travel survey to be included without making simplistic assumptions concerning charging. For the conventional vehicle data, distance is converted into energy expenditure using a conversion of $0.26 \mathrm{kWh} / \mathrm{mile}$ for rural areas and $0.35 \mathrm{kWh} /$ mile for urban areas based off analysis of the consumption of a Nissan Leaf in [50].

\subsubsection{Controlled Charging}

The perfect controlled charging scenario is calculated using an optimization problem. The objective is to flatten the total load profile, given knowledge of the existing electricity demand. It was assumed that only uni-directional charging was available at a maximum rate of $7 \mathrm{~kW}$ and with a $90 \%$ efficiency. Mathematically, the optimisation objective to be minimised can be described as:

$$
f(x)=\sum_{t}\left(d_{t}+\sum_{i} x_{t, i}\right)^{2},
$$

where $x_{t, i}$ represents the charging power of vehicle $i$ during time period $t$, and $d_{t}$ represents the existing electricity demand at that time. Each vehicle's charging profile must satisfy the following constraints:

$$
\begin{gathered}
\eta_{c} \sum_{t} \Delta t x_{t, i}=E_{i}, \\
0 \leq x_{t, i} \leq P_{\max } \quad \forall t \in \mathbb{T}_{i},
\end{gathered}
$$

where $E_{i}$ is the energy required by the EV, $\Delta t$ is the length of a time period, $\eta_{c}$ is the charging efficiency, $P_{\max }$ is the rating of the charger, 
and $\mathbb{T}_{i}$ contains the set of times where the vehicle is available to charge. Therefore, (2a) ensures the vehicle receives the required SOC, and (2b) imposes the limits of the charger. The resulting problem takes the form of a quadratic program and can be solved using standard solvers such as cvxopt [51].

\subsection{Simulation construction}

This section describes the formulation of the simulations which will be carried out at the transmission level and distribution level.

\subsubsection{Generation \&5 Transmission Simulations}

For simulations at the generation and transmission level stochasticity is not considered; at this level of aggregation the variance in vehicle behaviour will be very low. Instead a single worst case day is modelled, where the peak demand is highest - for GB, this is the coldest winter day. The simulations are constructed using the travel survey data scaled to represent the total GB population. For the transmission case, a separate simulation was constructed for each grid supply point, where the vehicles based in areas serviced by that point were extracted from the data. Load flow and optimal dispatch for the transmission simulation were carried out in Pandapower [52].

\subsubsection{Distribution Simulations}

For the distribution network studies, the high level of variance means that stochastic analysis is necessary. Separate simulations were constructed for each local authority in the UK. This geographic resolution was chosen as a trade-off between fidelity of results, and the size of the travel survey data once broken down by area. For each location, the relevant test network was selected based on the local rural-urban classification. Then a Monte Carlo simulation was run by randomly adding scaled household demand and vehicle charging profiles from the described datasets. The household loads were scaled according to the area's domestic electricity demand, and the energy to be charged was scaled according to the local vehicle usage recorded in the travel survey. At each iteration of the simulation, the optimal charging profiles were calculated, then the demand, losses and voltages in the network are recorded under each charging regime. Bus voltages and network losses were calculated using power flow simulations run in OpenDSS [53].

Based on observations of real secondary transformer ratings in GB and the design criteria in [54], one of three transformer limits was assumed. For the smaller two networks a $315 \mathrm{kVA}$ transformer is assumed for locations where fewer than $20 \%$ of households were on Economy 7 meters, and $500 \mathrm{kVA}$ otherwise. For the larger network a $800 \mathrm{kVA}$ transformer was assumed.

\section{Results}

Here the GB power system is studied to determine its best and worst case operation with domestic charging of private electric vehicles. First, the transmission level and distribution level systems are considered separately. Then, the conflict between the system levels is investigated.

In each case three scenarios are quantified: 'No Charging' describes the situation before any EV charging is added, 'Uncontrolled' predicts the charging without any intervention, and 'Controlled' considers charging that is optimised to flatten the total load on the system in question. Two EV penetration levels are considered: 100\% and the projected regional penetration in 2030 .

\subsection{Transmission Level System}

The transmission system will be impacted by the aggregated charging of the national vehicle fleet. In terms of generation capacity, the overall national demand profile is of concern. However, for transmission line loading the location of demand relative to supply is also important.

Figure 3a shows the additional energy demand of EVs in each local authority in 2030 and under $100 \%$ penetration. The $100 \%$ scenario demonstrates that the energy demand of vehicles has significant spatial variability across GB. Comparison with the 2030 case demonstrates that some of the worst affected areas in the $100 \%$ case will be slow to adopt EVs. This means the heaviest loaded areas predicted for 2030 are not the 
same as those with $100 \%$ electrification. Figure $3 b$ shows the power demand profile on a cold winter weekday with uncontrolled and controlled charging. Uncontrolled charging of a $100 \%$ electric fleet would increase the peak demand (and hence required generation) by $8 \mathrm{GW}$, however controlling charging can completely mitigate this increase.

The estimated winter demand profiles are similar to those found in existing research; National Grid (the GB transmission system operator) estimates that in 2030 there will be an increase in peak demand of between 2.6 and $8.6 \mathrm{GW}$, depending on the level of smart charging and EV penetration [55].

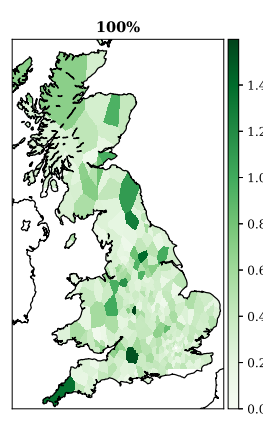

(a) Additional energy (GWh)

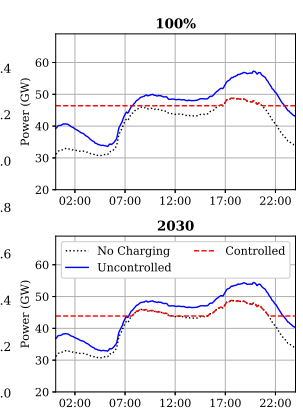

(b) Power demand
Figure 3: The energy and power demand from the UKs EV fleet in 2030 and with $100 \%$ penetration. (a) shows the location of the additional demand and (b) the uncontrolled/controlled power demand profile on a winter weekday.

Figure $4 \mathrm{a}$ shows the geographic distribution of peak demand in the $100 \%$ uncontrolled charging case. Each bubble represents a grid supply point whose size is proportional to the size of the peak load through that transformer. Figure $4 \mathrm{~b}$ shows the loading of the high voltage transmission lines resulting from this loading, as a percentage of their rated maximum current. None of the lines exceed their rated limit, and the majority of the lines are well within their design specifications. However, the security of the system is impacted. The UK has a target of $100 \%$ N-1 supply security, which means the demand could always be met if the single largest piece of infrastructure fails [56]. The markers show the lines whose loading would exceed their rating in the event of a failure of one of the other lines. The areas of concern are pri- marily in Scotland, where the system is at a lower voltage.

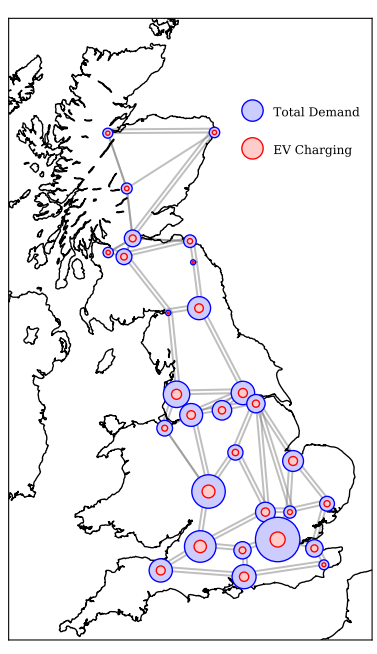

(a) Generation and loads

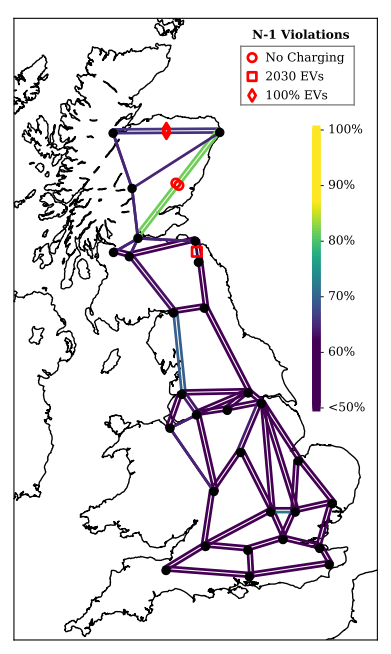

(b) Loading of lines
Figure 4: A reduced model of the UK transmission network under the peak load of uncontrolled charging; (a) shows the demand at each of the grid supply points, and the proportion of that which is from EVs; (b) shows the percentage of each line's rated loading being used.

Note that the effect of storage and HVDC interconnections have not been considered, so it is possible that the demand could still be met by utilising these resources.

\subsection{Distribution Level System}

In GB, residential low-voltage networks typically supply between 20 and 200 households with power. These networks are connected with transformers to the higher voltage distribution network. Additional load can cause a variety of problems; if the peak demand on the network surpasses the limit of the transformer it must be replaced, and resistive losses could result in unacceptably low voltages.

The impact of vehicle charging on the distribution network will vary significantly according to the local vehicles, households, and network structure. Therefore, here we consider the geographic variation in the likelihood of either of the network constraints being violated. First, we present the load profile, voltage drop, and losses for a single area, as an example to show the analysis we've 
completed for each local authority. Then, the estimated probabilities of a network constraint violations for each local authority in Great Britain are presented.

\subsubsection{Rochdale Case Study}

The simulated load, losses and voltages for Rochdale (a suburban area in Northern England) are shown in Fig. 5. Figure 5a shows the total load on the network under each charging scenario. The solid lines show the average load and the shaded area covers the $95 \%$ confidence interval. It can be seen that uncontrolled charging significantly increases the peak load, due to the coincidence of the charging and domestic peak demands. Controlled charging achieves a mostly flat load, and thus significantly reduces the peak compared to uncontrolled charging. The estimated transformer limit is $315 \mathrm{kVA}$, so a violation is probable in the uncontrolled charging scenario and possible with controlled charging.

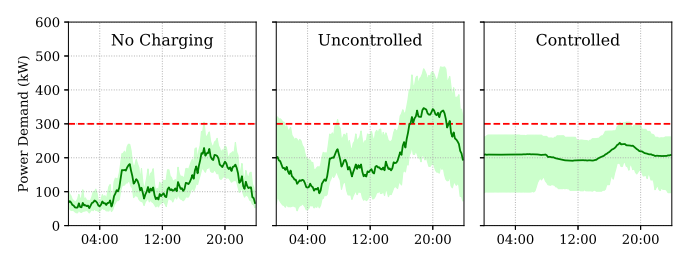

(a) Load Profile

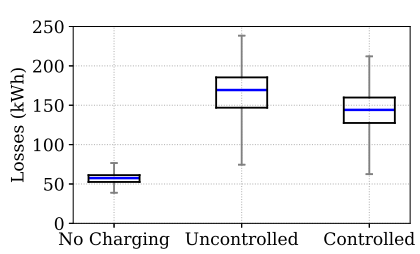

(b) Losses
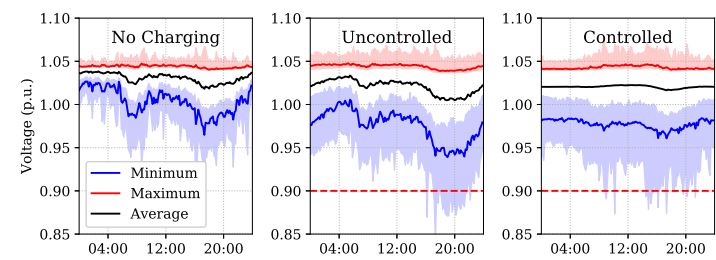

(c) Voltages

Figure 5: Load, losses, and voltages in an example network. Solid lines show average values and the shaded area covers the $95 \%$ confidence interval. The red dotted line shows the network constraints. In (b) the box covers $50 \%$ of the values, and the whiskers show the total range.
The losses associated with these loads are shown in Figure 5b, where the blue line shows the median loss, the box covers the interquartile range, and the whiskers the total range. The units shown here are $\mathrm{kWh}$, it should be noted that $1 \mathrm{kWh}=3.6 \times 10^{6} \mathrm{~J}$. In this case, uncontrolled charging triples the resistive losses in the network compared to the case without charging. Some of this increase can be avoided by controlling charging, however the increase in load means that increased losses are unavoidable. Resistive losses cause voltage drops across the network, so a voltage reduction can be expected under all charging scenarios. Safe system operation requires that the network voltage stay between $\pm 10 \%$ of the unitary voltage [9]. Therefore, voltage violations are possible under uncontrolled charging, but unlikely with controlled charging.

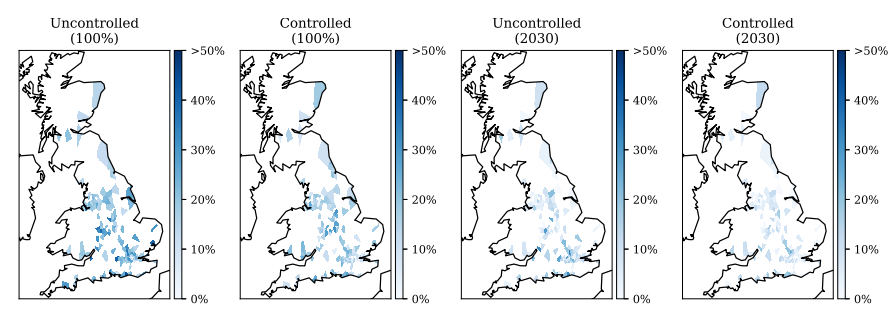

(a) Increased Probability of Voltage Violation (\%)

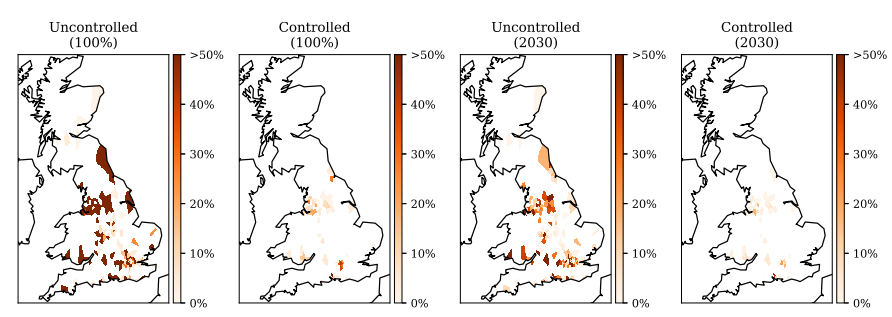

(b) Increased Probability of Transformer Violation (\%)

Figure 6: The increased likelihood of violating distribution network constraints in the UK, for charging of a $100 \% \mathrm{EV}$ fleet and in 2030 .

\subsubsection{Geographic Variation}

It is expected that the impact of charging on networks will vary significantly across the country. The probability of either a voltage or transformer violation occurring in each local authority is shown in Fig. 6, for both $100 \%$ penetration and projected in 2030 . EV sales are currently very unevenly distributed across GB, so some areas are 
likely to see a large penetration of EVs much earlier than others. Figure 7a shows the percentage of networks expected to experience each type of violation as time increases. Under uncontrolled charging transformer violations are more common than voltage violations, however with controlled charging the opposite is true.
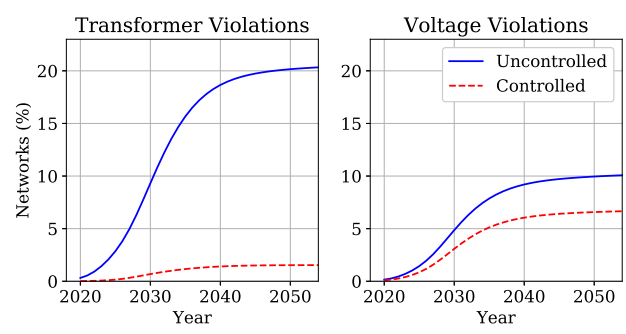

(a) Percentage of networks expected to have violations

\begin{tabular}{llll}
\hline \multicolumn{2}{c}{ Transformer } & \multicolumn{2}{c}{ Voltage } \\
\hline Stockton & $91 \%$ & Reading & $51 \%$ \\
Eastleigh & $90 \%$ & Worthing & $49 \%$ \\
Cannock & $89 \%$ & Sandwell & $47 \%$ \\
Dudley & $89 \%$ & Bristol & $46 \%$ \\
\hline
\end{tabular}

(b) Worst affected in $100 \%$

\begin{tabular}{llll}
\hline \multicolumn{2}{c}{ Transformer } & \multicolumn{2}{c}{ Voltage } \\
\hline Watford & $84 \%$ & Watford & $37 \%$ \\
Slough & $79 \%$ & Worthing & $33 \%$ \\
Gloucester & $75 \%$ & Gloucester & $33 \%$ \\
Swindon & $74 \%$ & Birmingham & $29 \%$ \\
\hline
\end{tabular}

(c) Worst affected in 2030

Figure 7: The percentage of networks estimated to experience each type of violation: (a) across all networks, as time increases, (b) and (c) in the worst affected areas.

In [57] it is estimated that $33 \%$ of GB distribution networks will require intervention as a result of EV charging, so these aggregated results are consistent with previous estimates. It should be noted that the $33 \%$ figure was not broken down by type or location of failure.

The local authorities most likely to experience either type of violation are displayed in Fig. 7b and $7 \mathrm{c}$. In the 2030 scenario, many of the same areas appear on both lists; this illustrates that, in the short term, the local penetration of EVs is the most important factor in determining whether reinforcements are necessary. In the $100 \%$ case there is a strong correlation between areas' trans- former and voltage violations - meaning areas likely to experience one, are also likely to experience the other. However, voltage violations were more likely in densely populated areas with large number of residents per household, while transformer violations were more likely in areas with high vehicle ownership.

As discussed in Section 2.3.2, for each area one of the three representative networks from Figure 1 is used for this analysis. It is worth noting that this will introduce some error into the results. The network used for the most urban areas (shown in Figure 1c) contained significantly more households than the sub-urban and rural networks, and this influences the type of constraint violations that are likely. Firstly, the larger number of consumers means that the diversifying affect between consumers is more significant, meaning extreme peaks in network load are less likely. Secondly, the electrical distance between the substation and furthest network is large, meaning significant voltage drop across the network could be expected. These combined factors mean that this network is less likely to experience transformer overloads and more likely to experience voltage violations. Therefore, the higher incidence of voltage violations observed in densely populated areas is at least partially related to the network structure. In fact, the four areas most likely to have voltage violations all use the urban network in Figure 1c. However, there were many areas which used this network where voltage violations were not encountered.

The differences between the rural and suburban networks are less obvious. The rural network has a slightly smaller number of consumers than are less regularly distributed along the network, but the effect this has will depend on the applied loads. In general, areas where transformer overloads were forecast were more likely to use one of these networks. However, rural and suburban areas have a significantly higher average travel distance than urban areas, so this is more likely to be an effect of the applied load. 


\subsection{Conflict between systems}

It is not possible to flatten load at both the distribution and transmission levels, due to the nonflat industrial load supplied by the higher voltage network. Therefore, the implications of controlling charging at each system level on the other level must be understood. Figure 8a shows the national load profile where charging is controlled at either the transmission or distribution level. When load is controlled at the distribution level the total load in residential networks is flattened, such that the values of $d_{t}$ in (1) represent the sum of the household load in the local network. This control scheme directly minimises the probability that the local transformer becomes overloaded. When load is controlled at the transmission level, the national demand is flattened, such that $d_{t}$ represents the national demand profile. This has the effect of minimising the national peak demand, and hence the required generation capacity.

Flattening load in residential distribution networks results in a $6 \mathrm{GW}$ increase in peak demand - almost comparable to that seen with uncontrolled charging. Alternatively, Figure 8b shows that purely flattening load at the transmission level results in $19 \%$ of residential networks requiring upgrades, compared with only $9 \%$ when flattening at the distribution level. However, it is possible distribution constraint violations could be reduced while maintaining an optimal load at the transmission level.

From Figure 6 it can be seen that many areas will not suffer constraint violations under any charging scenario, meaning that peak demand in these networks can withstand an increased load without upgrades. Therefore, an alternative controlled charging scenario was investigated where networks are ordered from most to least constrained, and allocated charging so as to flatten national load without violating their constraints. The most constrained networks will still be controlled to flatten the load at the distribution level. The least constrained networks offset the load in other networks in order to minimise national peak demand. Figure 8c shows the resultant national demand profile, broken down into industrial, domestic, and charging load. This profile achieves

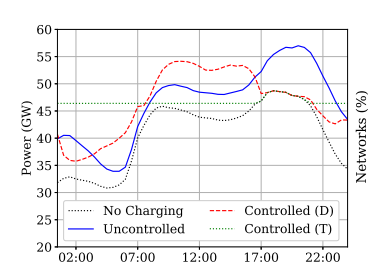

(a) National demand
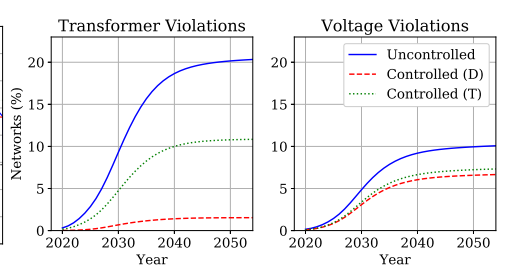

(b) Constraint violations

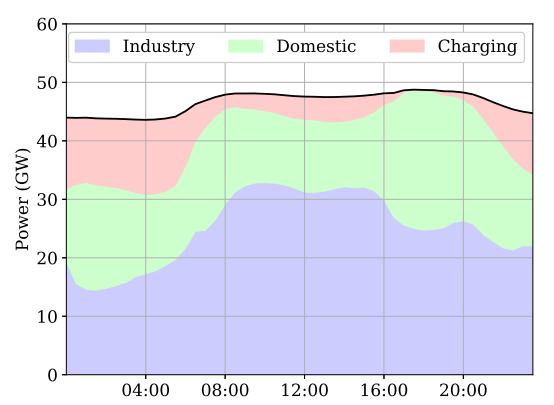

(c) National demand under $100 \%$ controlled charging without additional constraint violations

Figure 8: The implications of optimising charging for either the distribution (D) or transmission (T) system, and a national demand profile that heuristically optimises both systems, broken down by type of load.

an near-optimal transmission level load without increasing distribution network constraints. This suggests that smart charging, if appropriately implemented, could protect the GB system at both the transmission and distribution levels simultaneously.

\section{Discussion}

For the GB system, at the transmission level uncontrolled residential charging of a $100 \% \mathrm{EV}$ fleet would result in an $8 \mathrm{GW}$ increase in peak demand, and an increase in N-1 transmission violations. At the distribution level $28 \%$ of low voltage distribution networks would require upgrades. Transformer overloads were twice as likely as under-voltages. The low voltage networks most likely to experience problems were those where private vehicles were the primary mode of commuting, vehicle ownership was above average, and existing electricity demand was high. With a $100 \%$ penetration of EVs the worst hit areas were commuting towns to the west of London, and cities in the north of England. However, in the 
2030 projected scenario the local penetration of EVs had a much greater significance than the local travel behaviour.

Using smart charging to flatten total load can significantly reduce this impact. Flattening load at the transmission level completely mitigates the increase in peak demand, and flattening at the distribution reduces the percentage of networks with constraint violations to $9 \%$. However, flattening load at the residential network level will not flatten demand at the national level (and viceversa), due to the non-flat industrial load that is connected to the higher voltage network. Flattening load at the distribution level would result in a $6 \mathrm{GW}$ increase in national demand, while exclusively flattening load at the transmission level would mean an $19 \%$ of residential networks requiring upgrades, compared with only $9 \%$ with flattening at the distribution level.

Given that voltage violations appear to be less successfully mitigated than transformer overloads, it is likely that DNOs will still need to upgrade many networks. There are several methods of reducing the voltage drop along a network including: changing the transformer impedance, adding capacitor banks or voltage regulators, reconductoring cables, and reconfiguration of the network. The appropriate intervention will need to be decided on a case-by-case basis.

It was noted that many residential networks have oversized transformers, likely due to limited sizes and/or difficulty in estimating demand. This enables the additional flexibility in over-specified networks to be used to flatten load nationally without increasing constraint violations in residential networks. It was demonstrated that using an appropriate smart charging strategy, it is possible to eliminate the need for new transmission level generation infrastructure, while simultaneously achieving the same performance at the distribution system level as a strategy that exclusively flattened loads locally.

There are several areas for further work which could be explored. First, while it has been demonstrated that it is possible to optimise EV charging at the generation level without increasing distribution constraint violations, an applicable smart charging algorithm has not been developed here. The algorithm deployed in Section 3.3 merely demonstrated that a solution exists. In order to be practically applied, such an algorithm would need to schedule individual vehicle charging without relying on perfect forecasts of vehicle use. This is challenging because considering both the local and national problems simultaneously requires a large number of variables.

Second, an increase in distributed renewable generation has not been considered here. Local renewable energy generation can reduce the loads carried by the transmission system, but may cause local voltage issues in the distribution network. It is possible that EV smart charging could be used to offset voltage rise issues. Therefore, the simultaneous propagation of distribution generation and electric vehicle charging could be of great benefit to the system, and needs to be considered.

Third, an assessment of the impact of these charging regimes on system cost could be completed. The additional power demand created by EV charging has the potential to raise costs to the consumer - given the increase in system losses and national peak demand. However, the implementation of smart charging has the potential to create a more stable and efficient electricity system, resulting in a lower costs. HVDC interconnections could play an important role in achieving low system cost - particularly given the increasing role of renewable intermittent generation. Therefore, a cost analysis including an economic dispatch model would be an interesting area for further work.

\section{Conclusions}

This paper quantifies the impact that electric vehicle charging will have on transmission and distribution systems, and investigates how far smart charging can be used to reduce the impact on both systems.

Unlike previous analysis, spatial heterogeneity is incorporated into modelling of vehicle use, electricity demand, and network structure. Additionally, the conflict between the constraints of the transmission and distribution systems is quan- 
tified, and the degree to which both systems can be protected simultaneously is investigated

The numeric results presented in this paper are specific to Great Britain's system. However, some more general conclusions can be made. It is likely that vehicle use, electricity demand, and network structure will vary across a power system, and incorporating this variation is important in understanding how the system will be affected by electric vehicle charging. There is an inherent conflict between the transmission level and distribution level system when it comes to smart charging - it is not possible to flatten load both in residential networks and nationally. The degree to which it is possible to protect both systems will depend on a number of factors, including: the ratio of domestic to industrial load on the system, the amount of overhead in the existing distribution transformers, and the shape of the industrial load profile.

\section{Acknowledgements}

The authors would like to thank Jaguar Land Rover for their support in this research.

\section{References}

[1] Society of Motor Manufacturers \& Traders. Electric Vehicle Registration Data;. Accessed: 2019-04-05. https://www.smmt.co.uk/.

[2] UK. Future Energy Scenarios. National Grid; 2018.

[3] Paris Agreement; 2015-12-12. U.n.t.c. XXVII 7.d.

[4] Regen. Market Insight Series: Harnessing the Electric Vehicle Revolution. Regen; 2018.

[5] Verzijlbergh RA, Lukszo Z, Slootweg JG, Ilic MD. The impact of controlled electric vehicle charging on residential low voltage networks. In: 2011 International Conference on Networking, Sensing and Control, ICNSC 2011; 2011. p. 14-19.

[6] Darabi Z, Ferdowsi M. Aggregated impact of plugin hybrid electric vehicles on electricity demand profile. IEEE Transactions on Sustainable Energy. 2011;2(4):501-508.

[7] Van Vliet O, Brouwer AS, Kuramochi T, Van Den Broek M, Faaij A. Energy use, cost and CO2 emissions of electric cars. Journal of Power Sources. 2011;196(4):2298-2310.

[8] Quirós-Tortós J, Ochoa L, Butler T. How Electric Vehicles and the Grid Work Together: Lessons Learned from One of the Largest Electric Vehicle Trials in the World. IEEE Access. 2018;p. 64-76.
[9] British Standard Institute. Voltage characteristics of electricity supplied by public electricity networks; 2015 .

[10] Simma A, Axhausen KW. Interactions between travel behaviour, accessibility and personal characteristics: The case of the Upper Austria Region. Working Paper/IVT. 2003;182.

[11] Huebner GM, Hamilton I, Chalabi Z, Shipworth D, Oreszczyn T. Explaining domestic energy consumption - The comparative contribution of building factors, socio-demographics, behaviours and attitudes. Applied Energy. 2015;159:589 - 600. Available from: http://www.sciencedirect.com/science/ article/pii/S0306261915010983.

[12] Corpuz G. Public transport or private vehicle: factors that impact on mode choice;. .

[13] Piras A, Germond A. Local linear correlation analysis with the SOM. Neurocomputing. 1998;21(1):79 - 90. Available from: http://www.sciencedirect.com/ science/article/pii/S0925231298000332.

[14] Grahn P, Alvehag K, Soder L. PHEV utilization model considering type-of-trip and recharging flexibility. IEEE Transactions on Smart Grid. 2014;5(1):139148.

[15] Vagropoulos SI, Balaskas GA, Bakirtzis AG. An Investigation of Plug-In Electric Vehicle Charging Impact on Power Systems Scheduling and Energy Costs. Power Systems, IEEE Transactions on. 2017;32(3).

[16] Muratori M. Impact of uncoordinated plug-in electric vehicle charging on residential power demand. Nature Energy. 2018;.

[17] Procopiou AT, Quirós-Tortós J, Ochoa LF. HPCBased Probabilistic Analysis of LV Networks With EVs: Impacts and Control. Smart Grid IEEE Transactions on. $2017 ; 8(3)$.

[18] Sortomme E, Hindi MM, MacPherson SDJ, Venkata SS. Coordinated charging of plug-in hybrid electric vehicles to minimize distribution system losses. IEEE Transactions on Smart Grid. 2011;2(1):186-193.

[19] Li M, Lenzen M, Keck F, McBain B, Rey-Lescure O, Li B, et al.. GIS-based probabilistic modeling of BEV charging load for Australia; 2018.

[20] Xu Y, Çolak S, Kara EC, Moura SJ, González MC. Planning for electric vehicle needs by coupling charging profiles with urban mobility. Nature Energy. 2018;

[21] UK Government Department for Business, Energy \& Industrial Strategy. Government funded electric car chargepoints to be smart by July 2019;. Accessed: 2019-04-16. https://www.gov.uk/government/news/ government-funded-electric-car-chargepoints-to-besmart-by-july-2019.

[22] Cheng AJ, Tarroja B, Shaffer B, Samuelsen S. Comparing the emissions benefits of centralized vs. decentralized electric vehicle smart charging approaches: A case study of the year 2030 California electric grid. 
Journal of Power Sources. 2018;401:175-185.

[23] Ramadan H, Ali A, Nour M, Farkas C. Smart Charging and Discharging of Plug-in Electric Vehicles for Peak Shaving and Valley Filling of the Grid Power; 2019. p. 735-739.

[24] Nafisi H, Agah SMM, Abyaneh HA, Abedi M. TwoStage Optimization Method for Energy Loss Minimization in Microgrid Based on Smart Power Management Scheme of PHEVs. IEEE Transactions on Smart Grid. 2016;7(3):1268-1276.

[25] Weckx S, Driesen J. Load Balancing with EV Chargers and PV Inverters in Unbalanced Distribution Grids. IEEE Transactions on Sustainable Energy. 2015;6(2):635-643.

[26] Clairand JM, Garcia JR, Bel CA, Sarmiento PP. A tariff system for electric vehicle smart charging to increase renewable energy sources use. vol. 2017January; 2017. p. 1-6.

[27] Nour M, Said SM, Ali A, Farkas C. Smart Charging of Electric Vehicles According to Electricity Price; 2019. p. $432-437$.

[28] Dahmane Y, Ghanes M, Chenouard R, Alvarado-Ruiz M. Decentralized control of electric vehicle smart charging for cost minimization considering temperature and battery health; 2019.

[29] Vagropoulos SI, Bakirtzis AG. Optimal bidding strategy for electric vehicle aggregators in electricity markets. IEEE Transactions on Power Systems. 2013;28(4):4031-4041.

[30] Wu F, Sioshansi R. A stochastic operational model for controlling electric vehicle charging to provide frequency regulation. Transportation Research Part D: Transport and Environment. 2019;67:475 490. Available from: http://www.sciencedirect.com/ science/article/pii/S1361920918304590.

[31] Morstyn T, Teytelboym A, McCulloch MD. Matching Markets with Contracts for Electric Vehicle Smart Charging. vol. August; 2018. .

[32] Clairand JM, Rodriguez-Garcia J, Alvarez-Bel C. Smart Charging for Electric Vehicle Aggregators Considering Users' Preferences. IEEE Access. 2018;6:54624-54635.

[33] Moon SK, Kim JO. Balanced charging strategies for electric vehicles on power systems. Applied Energy. 2017;

[34] Aravinthan V, Jewell W. Controlled electric vehicle charging for mitigating impacts on distribution assets. IEEE Transactions on Smart Grid. 2015;6(2):999-1009.

[35] World Energy Council. WORLD ENERGY TRILEMMA INDEX; 2019. https://www. worldenergy.org/publications/entry/world-energytrilemma-index-2019.

[36] My Electric Avenue; http://myelectricavenue.info/.

[37] Lepanjuuri K, Cornick P, Byron C, Templeton I, Hurn J. National Travel Survey: 2015 Report. De- partment for Transport; 2016.

[38] National Grid. Historic Demand Data; 2019. https: //www.nationalgrideso.com/.

[39] Elexon. Profile Classes;. https://www.elexon.co.uk/ operations-settlement/profiling/.

[40] UK Govt Department for Business, Energy \& Industrial Strategy. LSOA domestic electricity 2016; . https: //www.gov.uk/government/statistics/lower-andmiddle-super-output-areas-electricity-consumption.

[41] Northern PowerGrid. Customer-Led Network Revolution; 2014. http://www.networkrevolution.co.uk/.

[42] W A Bukhsh and Ken McKinnon. Network data of real transmission networks; 2013. https://www.maths.ed.ac.uk/optenergy/ NetworkData/reducedGB/.

[43] Ochoa L. Dissemination Document "Low Voltage Networks Models and Low Carbon Technology Profiles". University of Manchester; 2015.

[44] UK Drivers and Vehicle Licensing Agency. Vehicle Registrations; 2018. https://www.gov.uk/ government/collections/vehicles-statistics.

[45] Nieto MI, López FG, Cruz F. Performance analysis of technology using the $\mathrm{S}$ curve model: the case of digital signal processing (DSP) technologies; 1998.

[46] Richardson P, Flynn D, Keane A. Optimal charging of electric vehicles in low-voltage distribution systems. IEEE Transactions on Power Systems. 2012;27(1):268-279.

[47] Sundstrom O, Binding C. Flexible charging optimization for electric vehicles considering distribution grid constraints. IEEE Transactions on Smart Grid. 2012;3(1):26-37.

[48] Xydas E, Marmaras C, Cipcigan LM, Jenkins N, Carroll S, Barker M. A data-driven approach for characterising the charging demand of electric vehicles: A UK case study. Applied Energy. 2016;162:763-771.

[49] Crozier C, Morstyn T, McCulloch M. A Stochastic Model for Uncontrolled Charging of Electric Vehicles Using Cluster Analysis; arXiv-2770789.

[50] Crozier C, Apostolopoulou D, McCulloch M. Numerical Analysis of National Travel Data to Assess the Impact of UK Fleet Electrification. In: Proceedings of the 20th Power Systems Computation Conference; 2018 .

[51] Vandenberghe L, Anderson M, Dahl J. CVXOPT; Available from: https://http://cvxopt.org.

[52] Thurner L, Scheidler A, Schäfer F, Menke J, Dollichon J, Meier F, et al. pandapower: An Open-Source Python Tool for Convenient Modeling, Analysis, and Optimization of Electric Power Systems. IEEE Transactions on Power Systems. 2018 Nov;33(6):6510-6521.

[53] Institite EPR. The Open Distirbution System Simulator (OpenDSS); 2018. http://smartgrid.epri.com/ SimulationTool.aspx.

[54] Croucher D. Design and Planning: Framework for underground networks in UK Power Networks. UK 
Power Networks; 2011.

[55] UK. Future Energy Scenarios. National Grid; 2019.

[56] Ofgem. Statutory Security of Supply Report. UK Department of Business, Energy, and Industrial Strategy; 2017.

[57] Storer N. How will the growth of electric vehicles impact the grid? Energy World. 2017;11:32-34. 